\title{
Waiting times and decision-making behind acute plastic surgery referrals in the UK
}

\author{
Mark Gorman $^{1}$, Chris Lochrin ${ }^{1}$, Muhammad Adil Abbas Khan ${ }^{2}$, Fulvio Urso-Baiarda ${ }^{3}$ \\ 1. Canniesburn Plastic Surgery Unit, Glasgow Royal Infirmary, United Kingdom. 2. Castle Hill Hospital, Cottingham, United \\ Kingdom. 3. Pinderfields Hospital, Wakefield, United Kingdom
}

Correspondence: Fulvio Urso-Baiarda. Address: Pinderfields Hospital, Wakefield, United Kingdom. E-mail: fulvio@doctors.org.uk

Received: July 3, 2012

Accepted: November 15, 2012

Online Published: December 4, 2012

DOI : $10.5430 /$ jha.v2n1p68

URL: http://dx.doi.org/10.5430/jha.v2n1p68

\section{Abstract}

Over the past decade, Accident and Emergency (A\&E) departments have struggled to cope with doubled patient numbers against a background of increased time constraints, accelerated medical training and shorter working hours. This study aims to investigate the view that A\&E referrals to Plastic Surgery are not always clinically motivated and seek alternative explanations for referral behaviours.

Reason for referral, time taken to refer and review, patient transfer time and demographics were documented from 100 consecutive A\&E referral case notes to a single Plastic Surgery Unit, comparing ‘appropriate' and 'inappropriate' referrals statistically. Semi-structured interviews with A\&E staff using the framework analysis method of deductive analysis were scrutinised to explore factors determining referral.

$69 \%$ of referrals were defined as "appropriate”. Inappropriate referrals were referred significantly more quickly than appropriate ones, but review time was similar between groups. Interview themes analysis revealed junior doctor inexperience, resource constraints and unintended consequences of targets and protocols as major non-clinical determinants of referral.

A significant proportion of A\&E referrals to Plastic Surgery are inappropriate, quicker referrals are more likely to be inappropriate and non-clinical priorities may determine referral behavior. This study provides insights into these priorities and could guide changes in medical education, postgraduate training and junior doctor supervision.

\section{Key words}

Waiting time, Accident and emergency, Plastic surgery, Referral

\section{I ntroduction}

Over the last decade, Accident and Emergency (A\&E) attendances have almost doubled from 12 to 21 million in England alone, amidst concerns regarding the system's ability to cope ${ }^{[1]}$. Concurrently several policy changes have been implemented, including the four hour waiting time target in 1995. As a result long waits, the previous major criticism facing A\&Es, have significantly reduced ${ }^{[2]}$ but possibly to the detriment of other aspects of patient care, notably patient safety and the patient experience ${ }^{[3]}$. For instance, patient treatments may have been prioritised by proximity to the end of a four-hour wait rather than clinical urgency, or patients may be moved to holding areas and experience very long waits, 
rather than wait over four hours in A\&E but receive definitive care there. Following recognition of this by a Department of Health review ${ }^{[4]}$, there has been a shift towards quality indicators of care rather than merely efficiency.

Other changes to the medical landscape have included alterations to doctors' working hours and restructured training. The General Medical Council's 2011 report ${ }^{[5]}$ regarding training highlighted reduced working hours as a key obstacle to the acquisition of skills and the ability to deliver high quality care. A UK audit indicated that decreased clinical exposure in A\&E has adversely affected doctors' ability to diagnose and triage the breadth of A\&E presentations ${ }^{[6]}$. This issue may be most apparent for specialties receiving little medical school exposure, including Plastic and Maxillofacial Surgery ${ }^{[7,8]}$. Decreased knowledge regarding hand anatomy, necessary to examine, triage and refer Plastic Surgery patients competently, has been highlighted as a key area to be addressed within training ${ }^{[9]}$.

Our previous work showed a striking correlation between shorter A\&E Referral Time and decreased referral appropriateness (defined as the need for surgery, admission or senior review) ${ }^{[10]}$, while all patients were seen in an equal time frame by the Plastic Surgery team regardless of appropriateness. However the study's prospective methodology and lack of blinding to the Plastic Surgery team raised the possibility of bias.

The current study was conducted in two parts. Phase one addresses methodological concerns of the previous study by carrying out the study retrospectively at a different UK hospital. The second phase attempts to understand the reasons for referral behaviours by interviewing a representative sample of A\&E staff and systematically analyzing their responses.

\section{Methods}

\subsection{Phase 1}

One hundred consecutive A\&E referrals to a single Plastic Surgery Unit (Castle Hill Hospital) were retrospectively reviewed (December 2010-February 2011). Medical notes were retrieved and, using a standardised proforma, investigators recorded patient demographics, reason for referral, Referral Time (the time between arrival in A\&E and referral to Plastic Surgery), Review Time and Referral Appropriateness. Since the nearest A\&E was at a different hospital site, Review Times excluded patient Transfer Time (time between referral from A\&E and presentation at Plastic Surgery unit), unless referred by and reviewed at the local A\&E.

'Referral Appropriateness' was defined by whether referrals required any of: subsequent hospital admission; surgery; or senior review. All referrals were reviewed by the Junior doctor on call (Senior House Officer grade), and if further senior input was required to help decision-making the referral was considered appropriate, even if that advice was for conservative treatment. Thus the definition of an appropriate referral was relatively inclusive.

Statistics were performed using the Statistics Package for the Social Sciences (SPSS). 'Appropriate' and 'Inappropriate' referrals were compared statistically for Referral Time, Transfer Time and Review Time using the independent samples t-test. Additionally, after stratifying referrals by hour of the day, the proportion of inappropriate referrals per hour was compared with mean Referral Time and mean Review Time per hour by means of Pearson correlation.

\subsection{Phase 2}

Through interviews with A\&E staff conducted on a single day (March 2011), phase 2 of the study explored the reasons for the referral patterns observed in phase 1. A generic email invitation to the department requested seven participants (to match the varying proportions of grades within $A \& E$ ) with places secured based on first response. One Consultant, one Registrar (SpR), three Junior Doctors (also referred to as SHO in the transcripts) and two Nurse Practitioners (NP) undertook semi-structured interviews commencing with the opening statement: 
'In reviewing Plastic Surgery referrals from this department, we have observed a correlation between the time it takes to refer and the appropriateness of referral, specifically, less appropriate referrals were made more quickly.'

With an initial statement or pre-stated aim (elucidating factors affecting referral behaviour) interviews (average duration 20 minutes) employed the deductive approach ${ }^{[11]}$ : after the initial statement, open questions became more directed, permitting both emerging and assumed themes to be systematically investigated.

Recorded on portable devices, the audio was transcribed by an independent blinded third party. Subsequent analysis was based upon the 'framework analysis method', previously validated for use in deductive analysis ${ }^{[12]}$. This involves five stages: First, audio transcript familiarization allows key issues and themes to be outlined. This leads to the Identification of a thematic framework, where recurrent interview themes may be cross-referenced against a priori issues. Key emergent themes are then indexed under numerical codes with their associated evidence and supporting statements noted. The indexed areas are charted and the transcript data patterns rearranged logically into main headings and sub-themes (see table 3 - results). Once mapped, themes may be defined and analyzed. Associations and links are explored between differing theme typologies and interpretations targeted towards the original research objectives.

\section{Results}

\subsection{Phase 1}

Of the 100 records reviewed, 27 were excluded due to insufficient documentation (Review or Referral Times). Patient demographics showed the majority of referrals were adult males (81.8\% male, mean age 39.6 years, 92.3\% aged 16 or older). Most referrals were for upper limb injury, of which half were finger injuries. Remaining referrals, for lower limb, head or trunk injuries, constituted only $11 \%$ of total referrals. (Table 1)

Table 1. Injury site distribution of A\&E Referrals to Plastic Surgery.

\begin{tabular}{ll}
\hline Injury Site & $\%$ \\
\hline Upper Limb & 89.1 \\
- Finger tip & 17.5 \\
- Finger & 50.9 \\
- Hand & 8.8 \\
- Wrist & 10.5 \\
- Forearm & 5.3 \\
- NOS & 7 \\
Lower Limb & 6.3 \\
Head/face & 3.1 \\
Trunk & 1.6 \\
\hline
\end{tabular}

The most common treatment outcomes were conservative management or wound exploration with no identifiable deep injury (both 22.7\%), followed by the management of infected or contaminated wounds (particularly animal or human bites) and extensor tendon repair.

The mean Referral Time was 1:53 hours, the longest Referral Time being 7:22 hours. The mean Review time was 0:58 hours, the longest being 6:10 hours. The longest Transfer time was 12:06 hours for an overnight referral asked to attend the following day.

69.2\% of referrals were defined as Appropriate (needing admission, surgery or senior review). The mean Transfer Time for Appropriate referrals was shorter than that for Inappropriate referrals (1:35 hours vs. 2:36 hours, $p=0.046$,), and 
Referral Time for Inappropriate referrals was significantly shorter than for Appropriate ones (1:09 hours vs. 2:13 hours, $p=0.015$ independent samples t-test). The Review time was similar for both referral types ( $0: 55$ vs. 1:04 hours, $p=0.695$ ).

Table 2. Referral and Review Times for children vs. adults and for appropriate vs. inappropriate referrals.

\begin{tabular}{llll}
\hline & & $\begin{array}{l}\text { Mean Referral Time/hrs } \\
\text { (Range, SD) }\end{array}$ & $\begin{array}{l}\text { Mean Review Time/hrs } \\
\text { (Range, SD) }\end{array}$ \\
\hline Age & Child & $1: 52(00: 15-3: 07$, SD 1:06) & $2: 48(0: 12-6: 10$, SD 2:28) \\
& Adult & $1: 55(0: 02-7: 22$, SD 1:37) & $0: 48(0: 00-3: 10$, SD 0:41) \\
Appropriateness & Appropriate & $2: 13(0: 10-7: 22$, SD 1:35) & $0: 55(0: 00-6: 10$, SD 1:11) \\
& Inappropriate & $1: 09(0: 02-5: 38$, SD 1:22) & $1: 04(0: 00-2: 05$, SD 0:37) \\
\hline
\end{tabular}

Review Time was reasonably consistent throughout the day except for a peak between 18:00 and 18:59. There were marked fluctuations in Referral Time throughout the day, referrals occurring most rapidly between the hours of 07:00-07:59, 17:00-17:59 and 19:00-19:59. These time points coincided with three of the four time periods when the Plastic Surgery department received the lowest proportion of Appropriate referrals. There was no statistically significant correlation between the proportion of Appropriate referrals per each hour period and either the mean Referral Tine or mean Review Time for the corresponding hour.

There was no significant difference in mean Referral Time between adults and children (1:55 vs. 1:52 hours, $p=0.942$, independent samples t-test). However, it took the Plastic Surgery team significantly longer to review children than adults (2:48 vs. 0:48 hours, $p<0.1)$.

\subsection{Results \& themes analysis - Phase 2}

Table 3. Emergent Themes and Sub-themes drawn from the data collection

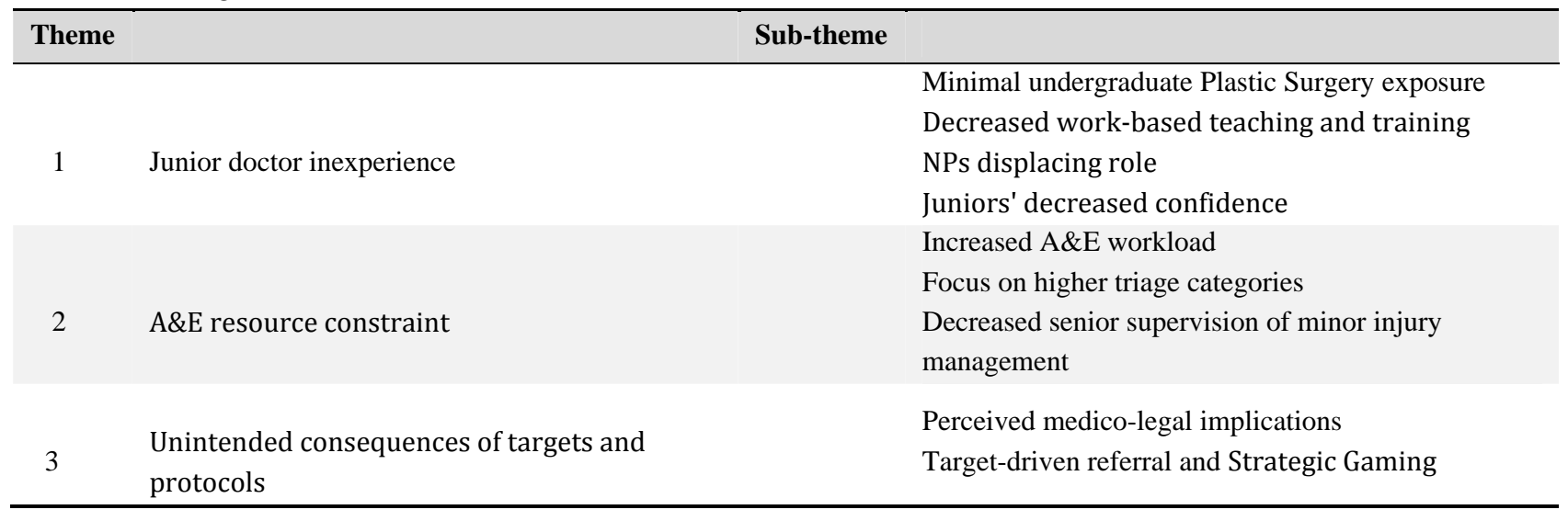

\section{Staff member key for quotes:}

\section{$\mathrm{C}=$ Consultant, $\mathrm{S}=\mathrm{SpR}, \mathrm{J}=$ Junior doctor and NP $=$ Nurse Practioner}

\subsubsection{J unior doctor inexperience:}

All grades of staff highlighted junior doctors' decreasing levels of experience:

C "Juniors coming through have increasingly less experience compared with previous trainees"

S “the SHO's have less clinical acumen than we had ...they don't get the same exposure.”

Minimal undergraduate Plastic Surgery exposure was repeatedly highlighted by the Junior doctors: 
J “There's nothing in med[ical] school regarding Plastics."

This contrasted with exposure to other specialities:

J "everybody knows a bit of General or Ortho[paedic Surgery]."

All grades voiced concerns regarding the effects of junior doctors' decreased exposure, stressed particularly by the NPs: NP “..It's knowing basic surface anatomy, signs and any red flag symptoms, which a lot of people don't know, [such as] flexor sheath signs and vascular compromise in fingers. Unless it's everyday routine to you, people are not confident”.

Decreased work-based teaching and training was felt by all to be a barrier to addressing these issues. Junior doctors reported receiving no formal teaching in Plastic Surgery:

J "Plastics? We don't get any teaching."

The SpRs noted the cessation of A\&E training sessions previously run by both Plastic and Orthopaedic Hand Surgeons. Linked to other sub-themes (resource constraint and senior supervision), the SpR's acknowledged a lack of training provision regarding Plastic Surgery:

S "I would like to teach more. When the opportunities arise on the job I tend to focus on resusc... - not Plastics!"

C "Formal introductory training sessions are already set-up, however this includes very little Plastic Surgery, which does need addressing."

In contrast NP work-based training was mandatory within the department. NPs displacing role [of trainees] was widely acknowledged:

NP "we tend to see more of the lacerations and wounds and the hand injuries."

J "All straightforward wounds or straightforward fractures, the Nurse Practitioners take them.”

An associated decrease of junior doctor confidence was mentioned recurrently:

J "You don't want to be hanging around doing stuff where really you think, well, I'm not an expert in it, and really plastics should probably examine it properly.”

The SHO's felt generally unsure regarding how to examine Plastic Surgery patients and that these issues were not addressed due to:

J “embarrassment”, and a belief that most Plastic Surgery cases

J "were not generally that serious".

\subsubsection{A\&E resource constraint}

Senior grades frequently cited an increased A\&E workload:

C "Increased admissions are secondary to many factors: increased GP referrals, patients perceiving that they will be seen more quickly or more easily than by their own GP and of course an increasingly elderly population.”

S "GPs are doing less, more patients coming in, more referrals."

Without increased resources, doctors repeatedly emphasised a shift in A\&E’s focus on higher triage levels:

$\mathbf{S}$ "A\&E is becoming more of an acute service and aggressive resuscitation, rather than a minor injury service now."

The same grades felt that this change in focus and "limited resources" was leading to decreased senior supervision of junior's clinical work outside resuscitation, especially regarding minor injuries:

S “I’m increasingly stuck in resusc, making it difficult for me to watch over... junior colleagues."

S "Often if a junior has a problem in minors [injuries], I will refer them on to one of the NPs as I don't have the time to deal with basic wounds that are not life-threatening."

Both the NPs and junior doctors highlighted a paradox resulting from the perceived lack of senior supervision. Within the NP protocol:

NP "challenging cases are referred onto the SHOs."

Yet often when requesting help from the SpRs, the junior doctors reported: 
J "we are told by seniors to go back to NPs when unsure".

\subsubsection{Unintended consequences of targets and protocol driven referral}

Perceived medico-legal implications were cited by all grades as a significant factor affecting A\&E treatment and referral. NPs refer more challenging cases to junior doctors, as per department protocol, despite the knowledge that:

NP “..the SHO's often did not know what to do.. the blind leading the blind."

Following protocols to defend against potential litigation was a theme repeated by all:

J “you might think oh, well litigation, I want to be careful here.”

Fears of litigation were cited by all as having a direct or indirect effect on what was treated in A\&E and what was referred on:

S "Well it's also a medico-legal thing as well. You know, we have a specialty to deal with these things."

C “..face lacs to be done by Plastics.. potential litigation.”

The effects of target-driven referral and strategic gaming were repeatedly highlighted:

J "we are told how many we see [patients] and how many we should be seeing."

Both NPs and junior doctors reported instances where meeting referral targets were of prime importance, as opposed to the quality of referral:

NP "If patients are about to breach then the pressure is to get a speciality to accept the referral. Triage is rushed and I often don't have the time to examine properly."

J “Under pressure you don’t explore wounds, you just triage.”

A culture of strategic gaming was cited as an unintended consequence of such targets. Examples illustrated include patients being re-assigned to holding areas to avoid breaches:

NP “To stop the clock... you move them onto the ward without them being officially accepted by Plastics.”

Encouraged by senior nurse bed managers, A\&E staff rushes through referrals prior to staff change over periods:

J "When the bleep holders are looking to clear the boards before change over, they make sure everybody is pushing though referrals.”

\section{Discussion}

We hypothesised that priorities other than clinical need may influence A\&E referral behavior. Phase 1 of this study confirms our earlier work supporting such a view, in which referrals assessed as inappropriate had significantly shorter Referral Times than appropriate ones. One difference in outcome between this and the earlier study was that it took the Plastic Surgery team significantly longer to review children than adults. This resulted from the departmental setup at the hospital trust at which the current study took place, in which there was no facility to review paediatric patients except at a separate hospital site (thirty minutes’ drive from the Plastic Surgery department).

In both this and the previous study, our interpretation of the data is that to process patients more quickly, A\&E staff may sometimes inadequately distinguish between patients who do and do not require referral. The feelings and analysis of A\&E staff elicited in Phase 2 support this inference and provide insights into what motivates referral behaviour. By retrospectively revisiting the original study in phase 1 , concerns regarding potential study bias have been avoided. As both the original and repeat study have independently shown one third of all referrals to Plastic Surgery to be inappropriate, identifying the associated causes should provide insights that may improve a suboptimal aspect of patient management. 
Referral behavior is a complex multifactorial process and its investigation is methodologically difficult. A mixed quantitative and qualitative approach enabled us to do this most effectively. A simple definition of referral appropriateness is hard to conceive and the surrogate indices used (the need for surgery, admission or senior review) were imprecise. However, we chose to use objective reproducible criteria in preference to case-by-case judgments after the fact. Our impression on reviewing the study data was that the proportion of inappropriate referrals was probably underestimated. The two most common treatment outcomes for A\&E referrals were conservative treatment or wound exploration without any deep injury (22.7\%). Whilst not inappropriate per se, the trends suggest that minor injuries, previously treated appropriately in A\&E, are now more likely to be referred. This is in keeping with the findings of others ${ }^{[13]}$, and is congruent with the themes elicited in phase 2 of the study, where changes in trainee doctor experience, medico-legal considerations and the unintended consequences of target driven medicine are cited as key drivers.

Mayhew's ${ }^{[2]}$ observational study of referral patterns highlighted that certain referral types were commonly rushed through to comply with the 4-hour wait target. Two thirds of Plastic Surgery referrals in this study were for upper limb (specifically hand) injuries, which may be particularly susceptible to this. A lack of junior trainee experience emerged as a key theme undermining their ability to triage cases effectively, and a temptation simply to refer 'hands to plastics' was acknowledged. The problem may originate from the lack of exposure to Plastic Surgery at an undergraduate level. Only one third of UK medical schools include Plastic Surgery in their curriculum and most A\&E doctors will therefore have received no relevant formal education when they commence A\&E posts ${ }^{[14]}$. This scarcity of exposure contrasts with other areas of surgery (for example, General and Orthopaedic Surgery). Unfortunately such a gap is not addressed during A\&E induction or on the job teaching and training. Juniors reported receiving instruction in trauma and resuscitation; but no training in Plastic Surgery.

Concerns that Plastic Surgery's importance is overlooked due to confusion regarding its role have been voiced since McCormack in the 1960's ${ }^{[15]}$. Perceptions, such as Plastic Surgery being an 'indulgent' specialty, are hard to shake. A survey of UK medical students showed that $85 \%$ could not list five conditions treated by plastic surgeons, $93 \%$ felt that cosmetic surgery was the core component of the specialty and the majority felt it irrelevant to training ${ }^{[7]}$. A targeted final year teaching program transformed students' core knowledge and understanding of Plastic Surgery's role. Such educational interventions are necessary for students to progress into postgraduate employment with the minimum working knowledge required for safe practice.

The push to develop core Plastic Surgery knowledge in A\&E may also be inhibited by the increasing attitude taken towards minor injuries, many of which fall under the remit of Plastic Surgery. Decreased senior supervision regarding minor injuries emerged as a theme in our analysis, with acknowledgments that minor injuries are delegated to NPs and junior doctor supervision is a low priority. In the context of other emergent subthemes, such as increased A\&E workload, and attempts to use resources wisely, a focus on higher triage categories is understandable but may fail to recognise that certain Plastic Surgery cases managed in the minor injury unit require early recognition of signs to avoid permanent functional impairment (e.g. vascular compromise, flexor sheath infection, high pressure injection). The basic relevant anatomical knowledge required to triage such Plastic Surgery cases safely is missing from junior's armamentarium and has decreased significantly over the last decade ${ }^{[9]}$. A survey involving UK trainees revealed they feel most competent working with 'medical trolley patients' and least so with minor injury patients. In keeping with our findings, the authors attribute this to lack of teaching, experience in handling minor injuries and the negative impact of Nurse-led minor injury units $^{[16]}$.

The theme of NPs displacing role on training was acknowledged by all staff. The NP role was introduced in the 1990's to address shortfalls in A\&E Rota's (associated with doctors' recently decreased working hours) and reduce waiting times. The NP role was partly seen to formalise a long-standing situation where senior nurses mentored junior doctors. However, mentorship did not form part of the NPs eventual role and appears to have been degraded by service demands placed on 
them. As cited in Tye's 1997 review ${ }^{[17]}$, The Royal College of Nurses (1992) definition of the NP role mirrors what should be expected of junior doctors, and with greater consideration, their impact could be to complement rather than displace:

"An [E]NP is an Accident and Emergency nurse who has a sound nursing practice base in all aspects of Accident and Emergency nursing, with formal post-basic education in holistic assessment, [and] physical diagnosis”.

Other unintended consequences, relating to target driven referral and strategic gaming, emerged as themes from our analysis. Investigating unintentional effects of targets, the Sheffield Medical Care Research Unit concluded that many emergency staff supported some benefits of the four-hour target, but the heavy financial penalties for breaches led to strategic 'gaming', such as a "spike" in activity during the last 20 minutes of the four hours. As the four-hour target has tightened the 'activity spike' has become more prevalent, affecting $30.7 \%$ of admissions and $10.5 \%$ of discharged patients in $2009^{[18]}$. In our study referral 'spikes' were noted at three times of the day preceding staff 'change over' times, where it was acknowledged that referrals were pushed through to 'clear the boards'. Other forms of strategic gaming included re-assigning patients to holding areas to avoid breaches. Governance issues arise as it becomes temporarily unclear who is responsible for patient care.

\section{Conclusion}

A significant proportion of referrals from A\&E to Plastic Surgery are inappropriate and early referrals are more likely to be inappropriate, consistent with the view that pressures and priorities other than clinical need sometimes motivate this behaviour. Resource constraint, a focus on higher triage categories and an underestimation of the potential seriousness of minor injury presentations have led to a situation where the levels of associated senior supervision and on the job training are insufficient. Many junior doctors currently lack the confidence and competence to manage routine cases that fall under the remit of Plastic Surgery. A lack of undergraduate exposure to Plastic Surgery and omission from most medical school curricula has led to levels of student awareness and basic working knowledge that are insufficient for safe future practise. This gap is not compensated for during A\&E jobs and training opportunities have become further restricted by factors including the NP role. Significant change is needed to address current situation. We hope such change may be informed by the insights of this study.

\section{References}

[1] BBC Today. (2012) 'We need fewer and better staffed A\&E units' [Internet]. Available from: http://news.bbc.co.uk/today/hi/today/newsid_9683000/9683014.stm [Accessed 10tth May 2012].

[2] Mayhew, L. Smith, D. Using queuing theory to analysed the Government's 4-hour time completion target in Accident \&Emergency Departments. Health Care Manag Sci. 2008; 11: 11-21. PMid:18390164 http://dx.doi.org/10.1007/s10729-007-9033-8

[3] Hamblin R. Publishing ‘quality’ measures: when it works and when it does not? Int J Qual Heaelth Care. 2007; 19(4): 183-186. PMid:17569682 http://dx.doi.org/10.1093/intqhc/mzm024

[4] Department of Health (2009). Accident and Emergency Episode Statistics [Internet]. Availible from: http://www.ic.nhs.uk/statistics-and-data-collections/hospital-care/accident-and-emergency-hospital-episode- statistics-hes [Accesssed 10th May 2012]

[5] General Medical Council (2011) The state of medical education and practice in the UK. General Medical Council, London.

[6] Bhandari S. A single-centre audit of junior doctors' diagnostic activity in medical admissions. J R Coll Physicians Edinb. 2009 Dec.; 39(4): 307-312. PMid:21152466 http://dx.doi.org/10.4997/JRCPE.2009.423

[7] Parikh, A. R., Clarke, A. \& Butler, P. E. Plastic surgery and the undergraduate medical school curriculum. Med Educ. 2006; 40: 476-7. PMid:16635148 http://dx.doi.org/10.1111/j.1365-2929.2006.02425.x

[8] Templer B, Amin K, Ahmed N, Fan K. Oral and maxillofacial surgery: the importance of undergraduate training for junior doctors in accident and emergency. Emergency medicine journal: EMJ. 2012. Epub 2012/04/27. 
[9] Dickson, J. K., Morris, G. \& Heron, M. The importance of hand anatomy in the accident and emergency department: assessment of hand anatomy knowledge in doctors in training. J Hand Surg Eur. 2009; 34: 682-4. PMid:19587072 http://dx.doi.org/10.1177/1753193409104947

[10] Urso-Baiarda F. An observational study of timing versus appropriateness of acute plastic surgery referrals in the UK. Int J Surg. 2009th ed. 2009 Dec.; 7(6): 539-542.

[11] Ritchie J, Spencer L. Qualitative data analysis for applied policy research. In Bryman A, Burgess R, eds. Analysing qualitative data. London: Routledge. 1993; 173-94.

[12] Pope C, Ziebland S, Mays N. Qualitative research in health care. Analysing qualitative data. BMJ. 2000; 320: 114-16. PMid:10625273 http://dx.doi.org/10.1136/bmj.320.7227.114

[13] Pinder RM, Urso-Baiarda F, Knight SL. Decades of change in plastic surgery training. J Plast Reconstr Aesthet Surg. 2010; 63(8): 662-3. PMid:20171947 http://dx.doi.org/10.1016/j.bjps.2010.01.037

[14] Burd A, Chiu T, McNaught C. Plastic surgery in the undergraduate curriculum: the importance of considering students' perceptions. British journal of plastic surgery. 2004; 57(8): 773-9.

[15] Mccormack, R. M. The role of plastic surgery in undergraduate surgical education. Plast Reconstr Surg Transplant Bull. 1960; 26: 309-11. PMid:13773825 http://dx.doi.org/10.1097/00006534-196009000-00006

[16] S J Croft, A Kuhrt, S Mason. Are today’s junior doctors confident in managing patients with minor injury? Emerg Med J. 2006; 23 (11): 867-868. PMid:17057141 http://dx.doi.org/10.1136/emj.2006.035246

[17] Christopher, C. The emergency nurse practitioner role in major accident and emergency departments: professional issues and the research agenda. Journal of Advanced Nursing. 1997; 26: 364-370. http://dx.doi.org/10.1046/j.1365-2648.1997.1997026364.x

[18] Mason S, Nicholl J, Locker T. Targets still lead care in emergency departments [Internet]. BMJ. 2010; 341: c3579. Available from: http://www.bmj.com/content/341/bmj.c3579.full [Accessed 2nd may 2012]. PMid:20605895 http://dx.doi.org/10.1136/bmj.c3579 